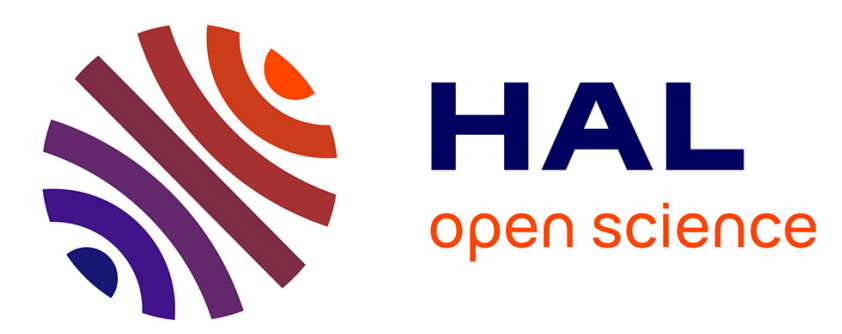

\title{
Effet d'un feedback informatif sur la prise de notes dans un environnement d'apprentissage informatisé
}

\author{
Sonia Mandin, Philippe Dessus, Benoît Lemaire
}

\section{To cite this version:}

Sonia Mandin, Philippe Dessus, Benoît Lemaire. Effet d'un feedback informatif sur la prise de notes dans un environnement d'apprentissage informatisé. STICEF (Sciences et Technologies de l'Information et de la Communication pour l'Éducation et la Formation), 2005, 12, pp.9-33. hal00323321

\section{HAL Id: hal-00323321 \\ https://hal.science/hal-00323321}

Submitted on 20 Sep 2008

HAL is a multi-disciplinary open access archive for the deposit and dissemination of scientific research documents, whether they are published or not. The documents may come from teaching and research institutions in France or abroad, or from public or private research centers.
L'archive ouverte pluridisciplinaire HAL, est destinée au dépôt et à la diffusion de documents scientifiques de niveau recherche, publiés ou non, émanant des établissements d'enseignement et de recherche français ou étrangers, des laboratoires publics ou privés. 


\section{Effet d'un feedback informatif sur la prise de notes dans un environnement d'apprentissage informatisé}

Sonia MANDIN, Philippe DESSUS [Laboratoire des Sciences de L'Education, Université Pierre Mendès France et IUFM de Grenoble]

Benoît LEMAIRE [Institut d'Informatique et de Mathématiques Appliquées de Grenoble (IMAG)]

- RÉSUMÉ : Cet article évalue les effets d'un feedback informatisé sur la qualité des notes prises pendant la lecture d'un cours. La prise de notes, en favorisant la mise en relation des informations lues avec nos connaissances, entraîne une réorganisation perturbante de ces dernières. Dans une démarche expérimentale, nous testons les effets d'un retour informatif concernant ce que les étudiants connaissent, sur le contenu de leurs notes (leur similarité avec le cours lu et leur cohérence interphrases). La similitude et la cohérence sont évaluées par un programme d'analyse sémantique latente (LSA) appliqué à un corpus textuel volumineux. Contrairement à nos attentes, nous n'observons pas d'effets dus au feedback mais des effets dus à la familiarité des participants avec le domaine du cours.

MOTS CLÉS : Prise de notes, acquisition de connaissances, analyse sémantique latente, environnement informatique d'apprentissage

ABSTRACT : This article assesses the computerized feedback effects due to the qualities of notes taken during the reading of a lesson. By inducing the connection between the existing knowledge and the information of a read lesson, the note-taking process entails a disturbing reorganization of knowledge. With an experimental approach, we describe the effects due to an inquiry feedback about the existing knowledge on the note's content (their similarity with the lesson text and their textual coherence). The similarity and the coherence are evaluated by the latent semantic analysis (LSA) applied to a large textual corpus. Despite our expectations, we don't find effects due to feedback but effects due to the familiarity of the participants with the domain of the lesson.

KEYWORDS : Note-taking, knowledge acquisition, latent semantic analysis, interactive learning environment

- Introduction

- La prise de notes dans un contexte d'apprentissage

- Des aides informatiques à la prise de notes

- Un feedback pour améliorer la prise de notes

- L'analyse sémantique latente

- Expérience

- Références 


\section{Introduction}

La formation ouverte à distance, l'apprentissage en ligne, les environnements informatiques d'apprentissage humain et le e-learning sont autant de notions qui, par ce qu'elles suggèrent, révèlent l'importance accordée aujourd'hui à l'apprentissage utilisant les nouvelles technologies de l'information et de la communication. Le nombre de dispositifs d'enseignement s'accroissant, les apprenants sont de plus en plus fréquemment amenés à étudier leurs cours face à un écran et à rédiger des textes directement en ligne éventuellement en prenant des notes. Face à cette situation, nous pouvons soit prendre le parti de comparer les nouvelles situations d'apprentissage aux situations plus traditionnelles de travail sur papier pour déterminer lesquelles des deux sont préférables, soit profiter de ce que la numérisation des productions offre comme perspectives d'analyse en temps réel qu'un autre contexte d'apprentissage ne permet pas. Nous nous inscrivons davantage dans cette seconde vision.

Dans le domaine de la prise de notes en contexte d'apprentissage, de nombreux travaux ont été réalisés. Certains sont centrés sur l'activité sur papier [CanivetA186], [Mayer87], [PeperMayer86], [PeverlyA103], alors que d'autres, souvent plus récents, sont centrés sur l'usage de la prise de notes avec un ordinateur [BlondelA100], [Beaufils01], [Blonde101]. Toutefois, dans ce dernier cas, l'objectif suivi est généralement celui de l'analyse des effets de telle ou telle interface de prise de notes. Ce sont des études davantage orientées sur l'ergonomie des applications que sur l'analyse des productions écrites.

Dans notre recherche expérimentale, nous postulons que les notes contiennent des traces des processus cognitifs effectués lors d'un apprentissage. Nous essayons donc d'expliquer l'acquisition de connaissances chez des étudiants par la description du contenu de leurs notes lors de la lecture d'un cours. Nous pensons qu'il s'agit d'un préalable qui peut permettre la conception d'environnements informatisés d'aide à l'apprentissage s'adaptant aux traitements cognitifs des utilisateurs. Cette approche utilise l'analyse sémantique latente, une méthode permettant notamment d'évaluer la similarité entre deux contenus textuels [FoltzA198], [LemaireDessus01] et une dimension de la cohérence d'un texte [FoltzA198], [LandauerDumais97]. Mais les étudiants ne disposent pas nécessairement tous des mêmes connaissances initiales. Certains sont plus familiers que d'autres avec le domaine traité, certains encore peuvent avoir mémorisé plus d'informations que d'autres lors de cours antérieurs. Ainsi, notre recherche nuance-t-elle la description du contenu des notes produites par la prise en compte de ce qui est acquis initialement et de ce qui ne l'est pas chez les participants. Il s'agit de leur faire identifier, par un feedback informatif sur leurs connaissances préalables du cours, ce qu'ils ne savent pas pour qu'ils puissent adapter leur comportement dans l'activité de prise de notes, et d'observer ainsi les modifications de l'effet du contenu des notes sur l'acquisition de connaissances. Après une présentation théorique de l'activité de prise de notes et de la méthode d'analyse sémantique utilisée pour le traitement informatique des traces produites, nous décrirons et discuterons l'expérience menée auprès d'étudiants de licence en sciences de l'éducation.

\section{La prise de notes dans un contexte d'apprentissage}

Plusieurs situations peuvent engendrer une prise de notes : par exemple, pour se rappeler les détails d'un rendez-vous, pour se souvenir d'une idée furtive, ou encore lors d'un apprentissage. Il est donc pertinent de mieux définir la prise de notes à la fois dans un contexte général et dans le contexte qui nous préoccupe, celui d'acquisition de connaissances par exposition à un cours.

\subsection{Qu'est-ce que la prise de notes?}

La prise de notes peut être associée tout autant à un pense-bête qu'à une technique d'apprentissage. Différentes définitions dans la littérature rappellent cette distinction. Certains auteurs font référence à la 
finalité de la prise de notes. Parmi eux, Reuter [Reuter94] la considère comme une démarche de synthèse ou de résumé. D'autres comme Simonet et Simonet [SimonetSimonet97] font référence à ses fonctions d'apprentissage. Ils la caractérisent comme une "démarche active d'enregistrement par écrit d'une information" dans le but de permettre des relectures ultérieures.

Oxford et Crookall [OxfordCrookall89] classent la prise de notes dans les stratégies cognitives d'apprentissage et la définissent comme technique qui entraîne la manipulation et la transformation directe d'informations. Elle facilite l'appropriation de connaissances [Dansereau85]. Romainville [Romainville93] est plus précis. Il distingue deux types de stratégies d'apprentissage: celles fondamentales qui agissent directement sur le traitement de l'information par des mécanismes cognitifs et celles de support qui produisent les conditions les plus favorables à l'apprentissage. Dans cette classification, la prise de notes peut alors être davantage assimilée à une stratégie fondamentale qu'à une stratégie de support : elle ne modifie pas physiquement l'environnement mais met en œuvre des processus cognitifs particuliers propres à toutes les activités rédactionnelles.

\subsection{Une activité rédactionnelle au service de l'apprentissage}

Piolat [Piolat01] désigne la prise de notes comme une activité dont les mécanismes cognitifs sont similaires à ceux de toutes activités rédactionnelles. Dans le modèle présenté par Hayes et Flower [Hayes98], la rédaction est contrainte par l'environnement de la tâche sociale (le destinataire des notes, la motivation de l'auteur, le texte déjà transcrit et le support d'écriture), les connaissances de l'auteur stockées dans la mémoire à long terme (MLT) et trois processus cognitifs faisant intervenir la mémoire de travail (interprétation, réflexion et production de texte). Kellogg [Kellogg98] affine la description de l'activité rédactionnelle en la reliant au modèle de la mémoire de travail (MDT) de Baddeley [Baddeley93]. Il distingue ainsi trois processus qui mobilisent la MDT :

- la formulation au cours de laquelle un tri des informations s'effectue en reliant les connaissances préalables stockées en MLT aux informations perçues lors de l'activité rédactionnelle ;

- l'exécution qui permet la transcription des représentations verbales ;

- le contrôle qui permet d'évaluer les dissonances entre le texte rédigé et le texte attendu.

Ces processus interviennent pendant une phase d'encodage des informations perçues. Mais l'activité de prise de notes ne s'arrête pas là. Les notes ont une durée de vie qui dépasse le temps de leur rédaction. Ainsi, ont-elles deux fonctions qui s'expriment à des moments différents : l'encodage et le stockage externe d'informations.

\subsection{Les deux fonctions de la prise de notes}

\subsubsection{L'encodage}

L'encodage est une première fonction de la prise de notes qui permet le traitement des informations auxquelles un individu est exposé. Pendant le traitement, le noteur rapproche les nouvelles informations perçues de ses connaissances préalablement acquises. A ce propos, Mayer [Mayer87] rappelle que l'apprentissage est favorisé par la réalisation de connexions internes et externes. Ainsi, les connexions internes permettent-elles de relier entre elles les différentes informations contenues dans un texte et les connexions externes de relier ces informations à ses propres connaissances.

D'autres travaux ont permis d'approfondir la connaissance de ces processus. Dans une première expérience sur des lycéens, Peper et Mayer [PeperMayer86] testent trois hypothèses d'attention (la prise de notes oblige à être attentif et à approfondir la compréhension), de distraction (les élèves devraient davantage se concentrer sur l'écriture que sur la lecture) et générative (les processus cognitifs propres à la prise de notes facilitent les connexions externes). Pour cela, ils présentent un texte aux élèves en demandant à certains de prendre des notes. Puis ils leur proposent quatre tests différents (tests de reconnaissance syntaxique, sémantique et factuelle, test d'application). Les résultats ne valident que la 
troisième hypothèse. D'une part, les scores obtenus au test d'application dans le groupe prenant des notes sont significativement supérieurs à ceux du groupe contrôle, signe de la réalisation d'un plus grand nombre d'inférences. D'autre part, les scores obtenus aux autres tests dans le groupe prenant des notes sont significativement inférieurs à ceux du groupe contrôle, signe d'une perturbation temporaire induite par une réorganisation des connaissances.

Une seconde expérience est menée par les mêmes chercheurs dans laquelle ils vérifient les prédictions d'un effet génératif, c'est-à-dire :

- les noteurs ont de meilleurs performances que les non-noteurs sur les tests d'application et inversement sur les autres tests ;

- cette différence est forte chez les étudiants dont les connaissances dans le domaine du texte exposé sont les plus importantes, et faible chez les autres ;

- les effets d'autres activités génératives sont semblables à ceux de la prise de notes.

Cette fois, les différents groupes sont constitués d'individus prenant des notes pendant la lecture ou après chaque section de texte, d'individus répondant à des questions après les sections ou n'y répondant pas, et d'individus d'un groupe contrôle ne prenant aucune note. Dans chacun des groupes, des personnes familières et non familières au thème sont présentes. En revanche, les tests sont identiques à ceux de la première étude. Les données recueillies dans cette expérience corroborent l'ensemble des hypothèses sur l'effet génératif de la prise de notes.

Plus récemment, Peverly, Brobst, Graham et Shaw [PeverlyA103] ont mené une expérience, proche de la nôtre, en prenant en considération à la fois les effets de la prise de notes et du niveau des connaissances préalables sur le texte étudié. Ils ont notamment recueilli les prédictions des étudiants sur leurs performances à trois moments-clefs : avant et après la lecture du texte exposé, ainsi qu'après la passation de tests (rappel libre, questions à choix multiples). Ils ont ainsi montré que la prise de notes permet aux étudiants d'améliorer leurs scores aux tests et de mieux juger de ce qu'ils connaissent. Par ailleurs, il semble également que le contenu des notes et les connaissances initiales sur le texte étudié soient des variables explicatives des résultats au test de rappel.

\subsubsection{Le stockage externe}

Mais la finalité de la prise de notes dans un contexte d'apprentissage ne se limite pas à l'amélioration de l'encodage des informations. Prendre des notes a également un intérêt à plus long terme, lors de la relecture des données transcrites. Alors que l'encodage s'effectue dès la première exposition à une source informative, la fonction de stockage externe ne peut intervenir sur l'apprentissage qu'ultérieurement ou plus précisément lorsque le noteur relit ses notes. Elles servent alors de support mémoriel.

Lors d'un cours oral, Canivet, Lecocq, Ledru et Sizaire [CanivetA186] montrent que la prise de notes ne nécessite pas une forte compréhension du discours mais qu'elle la favorise plus tard lors de la révision. Ils observent que les étudiants prennent davantage de notes quand le sujet n'est pas familier avec le domaine du discours, et que l'effet de la présence d'indices dans le texte source, inducteurs ou inhibiteurs de la prise de notes, est plus important. Ils émettent ainsi l'hypothèse que les indices permettent une sélection des informations en requérant un minimum d'effort cognitif et qu'un second traitement, plus profond, se fait au moment de la relecture.

Dans notre étude, nous nous sommes centrés uniquement sur la fonction d'encodage, qui favorise l'apprentissage en facilitant les connexions externes, et nous avons essayé de l'améliorer en réduisant l'effet perturbateur induit par une réorganisation des connaissances. Un dispositif informatique a donc été conçu pour guider la sélection des informations à transcrire et un programme d'analyse sémantique a été utilisé pour le traitement des notes recueillies. De nombreuses études concernent également le développement d'environnements informatisés pour la prise de notes. Ce qui suit en montre la diversité. 


\section{Des aides informatiques à la prise de notes}

Deux types d'études liées à la prise de notes peuvent être distingués selon leur objectif : d'une part, les études visant l'amélioration de la recherche documentaire (elles sont éloignées de notre problématique, mais permettent de découvrir différentes formes d'applications informatiques de prise de notes), et d'autre part, les études visant l'amélioration de l'apprentissage (elles sont plus proches de notre propre étude).

\subsection{Des aides à la prise de notes pour améliorer la recherche documentaire}

La recherche documentaire ne se limite pas à la consultation d'ouvrages en papier. Elle se fait également bien souvent avec un ordinateur. Dans un tel contexte, la prise de notes peut être améliorée en facilitant le stockage des informations trouvées. C'est ce que certains chercheurs montrent avec des applications comme ARI ou Eurisko. Nous les citons brièvement ici car bien que l'objectif de notre étude soit différent, elles contribuent à la construction d'une représentation d'un module de prise de notes sur ordinateur :

- ARI (Assistant à la Recherche d'Informations) : Blondel, Kempf et Schwob [BlondelAl00] font l'observation que les élèves de première scientifique, lors d'une recherche sur Internet, consultent un maximum de sites en prenant très peu de notes. Dans une étude suivante, Beaufils [Beaufils01] montrera que les élèves de quatrième et de seconde bénéficient d'une activité réflexive, préparatoire à la recherche documentaire, au cours de laquelle les élèves déterminent des mots-clefs, un premier plan pour une synthèse future et quelques requêtes. Partant de ces observations, ARI a été conçu pour faciliter la planification de l'activité lors d'une recherche d'informations sur Internet. Il permet ainsi de capturer des extraits de textes à partir de documents quelconques, de leur donner un titre, de leur ajouter un commentaire personnel, d'insérer leur adresse de stockage (en local ou en réseau) et de les classer dans une arborescence de dossiers [Beaufils01], [Blondel01], [BlondelA100]. ARI facilite donc l'encodage en assistant la prise de notes, et favorise la relecture en simplifiant l'accès ultérieur aux informations stockées.

- Prototype d'aide à la recherche d'information associé à Eurisko: Il est issu d'une étude basée sur le modèle cognitif EST de Rouet et Tricot [TricotA198] selon lequel la recherche d'informations dans un environnement documentaire complexe se déroule en trois étapes cycliques d'Evaluation du travail, de Sélection et de Traitement des informations trouvées. L'étude a un triple objectif : l'amélioration de la représentation d'une base de données, de la synthèse des résultats de recherches et de la conservation des informations trouvées. Pour cela, le prototype ajoute notamment un espace de travail individuel de prise de notes (notes personnelles, références et extraits de textes) à une base documentaire hypermédia sur la Grèce antique (Eurisko). Cet espace est affiché continuellement dans un cadre à l'écran et accessible rapidement. Il peut toutefois être masqué au gré de l'utilisateur. Les notes sont modifiables, regroupées en chapitres et classables selon divers critères [Beaufils01]. L'expérience est réalisée sur trois groupes (l'un avec la base Eurisko sans le prototype, les deux autres avec la base Eurisko associée au prototype) dans des classes de troisième et de première. Elle rend compte qu'un espace destiné à la prise de notes réduit les risques d'oubli et favorise l'attention.

\subsection{Des aides à la prise de notes pour améliorer l'apprentissage}

L'un des autres axes possibles de recherche est d'améliorer la prise de notes sur ordinateur pour favoriser l'apprentissage. Profitant des possibilités offertes par l'informatique, la plupart des études cherchent à valider une méthode spécifique de prise de notes sur ordinateur. Plus rares sont celles qui en testent la pertinence en comparant une prise de notes sur ordinateur à une prise de notes sur papier. Nous citerons toutefois l'étude de Gérouit, Roussey, Barbier et Piolat [GérouitA100]. Des élèves de 10-12 ans plus ou moins bons lecteurs rédigent un texte argumentatif à partir de notes transcrites au cours d'une recherche 
documentaire dans une base de données multimédia. L'accessibilité des informations et leur rétention sont évaluées à partir du texte produit et d'un questionnaire portant sur les impressions laissées par le dispositif. Pour cette expérience deux outils sont nécessaires :

- un site web multimédia adapté aux besoins (suppression des hypertextes pointant sur des sites externes, modification de certains contenus, insertion d'un sommaire en page d'accueil) et présenté sur un CDROM. Le site se décompose en huit rubriques possédant chacune des liens inter et intra rubriques.

- un logiciel de traitement de textes pour la prise de notes (WordPad) qui offre un minimum d'options de mise en page.

Durant cette expérience, un premier groupe a accès à la prise de notes via WordPad et un second via l'utilisation de papier/crayon. Deux limites sont imposées : une limite de temps dans la phase de recherche documentaire et dans la phase de rédaction ainsi qu'une limite d'espace pour la prise de notes. Les principales conclusions sont que les élèves écrivent moins avec l'outil papier/crayon, et que la pertinence des notes est plus faible chez les mauvais lecteurs. A propos de ces derniers, Gérouit et al. [GérouitA100] écrivent: "ils peuvent focaliser leur attention sur les processus de compréhension, sur le maintien en mémoire de travail du but de la recherche d'informations et sur l'évaluation des éléments sélectionnés".

Dans notre étude, nous travaillons sur des notes produites avec une organisation imposée minimale, comme dans celle de Gérouit et al [GérouitA100]. Cependant, nous ne nous inscrivons pas dans une étude comparative entre la prise de notes sur ordinateur et la prise de notes sur papier. Nous nous intéressons à l'effet de la prise de conscience de connaissances sur les notes produites et sur l'acquisition de connaissances par exposition à un cours. La prise de conscience est stimulée par un feedback.

\section{Un feedback pour améliorer la prise de notes}

Par définition, le feedback est un retour fourni à un sujet sur l'action qu'il vient de réaliser. Il prend la forme d'un résultat plus ou moins précis tel qu'une appréciation, un classement ou une réponse exacte. Le feedback a deux fonctions : la première, la plus évidente, est une fonction d'information, et la seconde, une fonction de motivation [Fenouillet01], [Leplat02]. Leplat [Leplat02] préfère le terme de "connaissance du résultat". Mais, utilisant le feedback essentiellement pour sa première fonction, nous préférerons utiliser ici les termes de "feedback informatif", également employés dans la littérature [Fenouillet01].

Pour qu'un comportement soit modifiable, le feedback doit succéder à une action. Dans la littérature, nous trouvons essentiellement des expériences réalisées sur des tâches précises et dans lesquelles le feedback a un caractère rétroactif [Bruillard97]. Une notion proche est alors celle de renforcement. Mais d'autres études détournent quelque peu l'utilisation du feedback: le but n'est plus de modifier l'action par correction d'erreurs, mais d'observer l'effet d'un feedback sur un processus cognitif. L'étude d'Olive et Piolat [OlivePiolat02] a été réalisée dans ce but. Ils étudient l'effet sur les processus d'écriture d'une absence de feedback visuel, autrement dit, de l'absence de la trace visible laissée par l'encre d'un crayon sur le papier quand nous écrivons. Ils concluent que le feedback visuel n'améliore que les processus d'exécution dans la tâche rédactionnelle. Dans notre propre étude, nous utilisons également le feedback d'une manière atypique. Il sert de retour informatif sur les connaissances préalables qu'un étudiant possède sur le texte présenté. Nous choisissons donc de le prodiguer avant la phase d'exposition au texte et d'en observer les effets sur l'acquisition de connaissances et sur le contenu des notes transcrites en cours de lecture.

Si l'analyse des résultats d'un questionnaire s'effectue classiquement, ce n'est pas le cas de l'analyse de contenu des notes produites. Aussi, nous avons donc recours à une analyse sémantique informatisée, l'analyse sémantique latente. 


\section{L'analyse sémantique latente}

L'une des façons d'observer les différents traitements cognitifs effectués par les noteurs est de se centrer sur le contenu des informations relevées. Deux indicateurs semblent alors pertinents : la cohérence des notes et leur similitude au cours exposé. LSA fournit une méthode pour rendre compte de ces indicateurs.

\subsection{Présentation d'une méthode informatique : l'analyse sémantique latente (LSA)}

LSA (Latent Semantic Analysis) est un modèle mathématique conçu par Landauer et Dumais [LandauerDumais97] permettant de décrire statistiquement le sens d'un mot à partir des contextes dans lesquels ce mot ou un autre sémantiquement proche apparait. Parmi les divers domaines dans lesquels LSA fut utilisé, nous trouvons celui de l'évaluation des connaissances d'un sujet à partir de ses productions écrites [RedhderA198], [WolfeA198]. Le programme reçoit en entrée un corpus de textes qu'il représente dans un espace vectoriel multidimensionnel et produit en sortie les résultats d'un traitement statistique. Les résultats correspondent à la proximité sémantique qui existe entre les différents éléments textuels (mots, phrases, paragraphes). Les calculs sont issus de la linguistique distributionnelle et basés sur les deux propositions suivantes [Landauer02], [LemaireDessus03] :

- le sens d'un mot peut être défini statistiquement à partir de l'ensemble des contextes dans lesquels il apparaît,

- le sens d'un mot peut être défini statistiquement à partir des occurrences des mots desquels il est luimême cooccurrent.

De plus, Kontostathis et Pottenger [KontostathisPottenger02] montrent que des cooccurrences d'ordre supérieur interviennent aussi. Si un terme $t_{1}$ est cooccurrent d'un terme $t_{2}$, le terme $t_{2}$ est cooccurrent d'un terme $t_{3}, \ldots$ et un terme $t_{n-1}$ est cooccurrent d'un terme $t_{n}$ alors, $t_{1}$ est lié à $t_{n}$. Leur étude sur cinq corpus différents (deux corpus de textes spécifiques, un corpus de mots et un corpus de phrases nominales) souligne que les paires de cooccurrences relevées ne dépassent pas le niveau 3, excepté pour le corpus de phrases nominales qui atteint des cooccurrences d'ordre 5. LSA rend compte de ces cooccurrences d'ordre supérieur à 2 .

Ainsi, chaque terme correspond-il à un vecteur. Un groupe de termes, tel qu'une phrase, est représenté par le vecteur somme des vecteurs des termes qui le composent et la proximité sémantique entre deux éléments est estimée par le cosinus de l'angle formé par leurs vecteurs respectifs. Cette méthode permet ainsi d'estimer notamment la compréhension d'un texte par une mesure de la cohérence interphrases [FoltzA198] et d'évaluer les connaissances contenues dans une production écrite par comparaison à des connaissances de référence [DessusA100]. Mais la pertinence des résultats repose avant tout sur l'input fourni au programme.

\subsection{L'importance du corpus}

Le corpus utilisé avec LSA joue le rôle de connaissances préalables. Il est donc important de le construire précautionneusement afin qu'il représente au mieux les connaissances réelles de la population étudiée. Deux critères doivent être pris en compte dans le choix d'un corpus : la quantité et le contenu des données.

Les performances de LSA dépendent d'un corpus volumineux. Dessus [Dessus00] montre que LSA est un modèle qui représente l'acquisition du vocabulaire. La proximité sémantique des mots évolue au fur et à mesure de l'exposition à des textes qui les contiennent et de manière différente selon leur importance dans le contexte (les termes dont l'importance varie au cours du texte ont une proximité sémantique entre eux généralement croissante et plus rarement décroissante, en revanche ceux correspondant à des notions centrales du texte ont une proximité sémantique à peu près constante). 
Par ailleurs, les performances de LSA dépendent aussi du contenu du corpus. Kurby, Wiemer-Hastings, Ganduri, Magilano, Millis et Mc Namara [KurbyA103] réalisent une expérience dont l'un des objectifs est de comparer les classements d'évaluateurs humains à ceux de LSA utilisé avec des corpus spécialisés et des corpus généraux. Les deux types de corpus sont construits à partir d'un même corpus comprenant des textes dans le domaine des sciences, qu'ils soient spécialisés ou non. Les corpus spécialisés sélectionnés diffèrent des corpus généraux par leur proportion plus importante de textes spécifiques. Les classements s'effectuent à partir d'explicitations à voix haute produites lors de la lecture d'un texte scientifique. Chaque phrase d'explicitation est comparée à trois types de termes indiquant ainsi si le lecteur réfère à la phrase lue (stratégie minimale), à ce qui précède (stratégie locale) ou à des connaissances du domaine (stratégie globale). Les auteurs concluent qu'un corpus spécifique permet un classement plus proche de celui d'humains. Ces résultats incitent à ne pas considérer uniquement un critère de taille dans la sélection de corpus. Il faut également qu'il soit approprié au domaine du texte de référence. C'est dans cette optique que nous avons construit un corpus particulier pour notre expérience.

Cependant, l'une des limites de LSA réside dans le fait que la construction de connaissances se fait à partir d'un corpus alors que les humains utilisent dans une tâche d'apprentissage non seulement le cours mais aussi des connaissances non décrites dans des textes. Glenberg et Robertson [GlenbergRobertson00] rappellent que, pour comprendre la signification d'un symbole abstrait, nous ne pouvons pas nous référer uniquement à d'autres symboles abstraits. Aussi, LSA ne peut pas expliquer toute la sémantique. Les auteurs le vérifient dans une première expérience où ils comparent les jugements de personnes sur des phrases (sont-elles sensées ? sont-elles imaginables ?) aux indices de proximité sémantique calculés par LSA entre la phrase initiale et une phrase testée puis entre les deux mots principaux des phrases testées. Dix-huit situations sont expérimentées, chaque situation correspondant à trois phrases : une cohérente avec les deux mots conceptuels principaux éloignés sémantiquement (afforded), une non cohérente (nonafforded) et enfin, une cohérente avec les deux mots conceptuels proches sémantiquement (related). Les résultats de cette expérience montrent que les personnes différencient chaque type de phrases alors que LSA ne le fait pas entre les phrases afforded et nonafforded. Une explication serait alors que les sujets répondent aux questions en tenant compte de leur expérience non linguistique. Les mêmes conclusions sont obtenues dans une seconde expérience relativement semblable. Enfin, une dernière étude introduit, dans les textes soumis aux sujets expérimentés, des verbes dont l'utilisation est nouvelle. Glenberg et Robertson [GlenbergRobertson00] estiment ainsi que l'homme peut comprendre un sens nouveau alors que LSA ne le peut pas. Cependant, Landauer [Landauer02] répond à cette objection en rappelant que parmi les phrases testées par Glenberg et Robertson, dix mots sont présents alors qu'ils ne figurent pas dans le corpus. Cela souligne, là encore, l'importance de ce dernier.

\subsection{La mesure de la cohérence textuelle}

Dans une étude de Lehman et Schraw [LehmanSchraw02] sur les effets sur la compréhension de la cohérence et de la pertinence d'un texte, la cohérence est définie comme une mesure par laquelle les segments de textes apparaissent liés structurellement entre eux et induisent un rapprochement avec les informations mémorisées. Par conséquent, deux types de cohérence sont distingués : la cohérence locale et la cohérence globale. La première représente le niveau d'ambiguïté entre deux phrases contiguës et la seconde est celle qui agit sur les inférences construites par le lecteur et les associations entre ses connaissances initiales et les informations lues pour améliorer la représentation de la situation décrite dans le texte.

Foltz, Kintsch et Landauer [FoltzA198] ont élaboré une méthode pour mesurer la cohérence textuelle avec LSA. Ils ont comparé la cohérence estimée par des techniques manuelles particulières à la cohérence estimée par LSA en calculant la moyenne des cosinus entre chaque phrase consécutive (valeurs comprises entre -1 et 1). Dans une première étude, Foltz, Kintsch et Landauer [FoltzA198] tentent de montrer que la compréhension d'un texte peut être prédite en mesurant sa cohérence avec LSA. Pour cela, ils comparent les résultats de sujets à des tests de compréhension à la cohérence estimée des textes proposés. Ces textes 
sont des déclinaisons d'un seul et même document (texte rendu cohérent par la méthode de Britton et Gulgoz (1991) [FoltzA198] $]^{1}$; texte clarifié en ordonnant les points importants et en supprimant les points superflus ; texte rendu moins lisible ; texte original). Conformément à ce qui était prédit, LSA désigne les deux premiers textes comme ceux qui sont estimés les plus cohérents. Les auteurs reprennent ainsi une méthode basée sur la définition de cohérence énoncée par van Dijk et Kintsch (1983) [LandauerDumais97]. Par ailleurs, dans l'expérience de Foltz, Kintsch et Landauer [FoltzA198], les sujets qui ont lu les textes les plus cohérents sont également ceux qui obtiennent les meilleurs scores en rappel libre, à un QCM testant des inférences et en efficience (rapport entre le nombre de propositions rappelées et le temps de lecture). Les auteurs identifient ainsi une forte corrélation entre la cohérence d'un texte et les caractéristiques mesurées de la compréhension.

\subsection{La mesure de la similarité de contenu}

Différents logiciels se basent sur LSA pour comparer les informations contenues dans une production écrite d'élèves à des connaissances de référence. Ces logiciels l'utilisent pour son aptitude à modéliser l'évaluation des connaissances. Différents types de scores sont ainsi déterminés.

Considérons tout d'abord le logiciel IEA (Intelligent Essay Assessor) de Foltz, Kintsch et Landauer [FoltzA198], conçu pour la notation de productions d'élèves. Il attribue un score dit gold standard issu de la comparaison entre une copie d'élève et une copie "idéale" mais aussi un score holistique, associant pour chaque copie d'élève, le score donné par un évaluateur humain à la copie de référence la plus proche sémantiquement. En revanche, dans l'application Apex [LemaireDessus01], les scores basés sur des comparaisons déterminent des scores de contenus (comparaisons des copies aux textes lus) et de plan (comparaison de chaque paragraphe rédigé à chaque notion étudiée). Dans la présente étude, nous déterminons la similarité de contenu par une comparaison sémantique d'un cours aux notes transcrites pendant sa lecture.

LSA, par les possibilités de mesures d'une dimension de la cohérence d'un texte et de la similitude de contenu entre deux documents, devient alors une méthode permettant une évaluation rapide et neutre des notes. Voici donc maintenant la description de l'expérience menée, suivie d'une discussion sur les résultats obtenus.

\section{Expérience}

\subsection{Problématique et hypothèses}

L'expérience que nous présentons consiste à faire lire un cours à des étudiants après avoir évalué leurs connaissances préalables. Nous observons ainsi l'effet d'un retour de cette évaluation sur la prise de notes et sur l'apprentissage. Comme nous l'avons déjà signalé, d'après Peper et Mayer [PeperMayer86], la prise de notes facilite l'apprentissage en favorisant les connexions externes. Toutefois, de moins bons résultats à des tests de reconnaissance et de rappel sont observés quand le lecteur note. Cette baisse est provoquée par une perturbation liée à la réorganisation des connaissances en mémoire. Ne pourrionsnous donc pas améliorer ces résultats en agissant sur les connexions externes ? L'idée expérimentée serait alors d'inciter les noteurs à relier les informations du texte, non pas à leurs connaissances acquises, mais à leurs connaissances déficitaires.

Différents courants de pensée sur l'apprentissage cherchent à améliorer les connaissances individuelles par l'utilisation d'un feedback. La conception béhavioriste skinnérienne qui veut que ce dernier soit utilisé comme un renforcement positif d'un comportement, s'oppose alors à la conception cognitiviste de Crowder qui présente le feedback comme un moyen de permettre à l'apprenant de se rendre compte de ses erreurs dans le but d'approfondir les points problématiques [Bruillard97]. En nous situant dans ce dernier courant, nous espérons améliorer l'acquisition de nouvelles connaissances par exposition à un 
texte en utilisant un feedback informatif sur l'exactitude des réponses aux questions posées. Ce retour simple et instantané permettrait à l'apprenant de prendre conscience que ce n'est plus l'intégralité du cours qui est à apprendre, mais seulement ses connaissances erronées ou incomplètes qui sont à corriger ou à compléter. Autrement dit, le feedback permettrait au lecteur de mieux se rendre compte des sections $\mathrm{du}$ texte les moins connues. Notre hypothèse est alors que le lecteur focaliserait davantage sa prise de notes sur ces sections, augmentant ainsi la similitude de contenu entre les notes et les sections méconnues $\mathrm{du}$ texte (similitude de contenu partiel). Par ailleurs, il organiserait ses notes autour d'un objectif clairement identifié, ce qui en augmenterait la cohérence textuelle interphrases.

\subsection{Méthode et traitements}

Les sujets. Quarante-quatre étudiants en licence de sciences de l'éducation de l'université Pierre-MendèsFrance (Grenoble) participent à l'expérience. Ils sont volontaires et reçoivent un crédit d'un demi-point pour leur participation. Parmi eux, certains suivent l'unité d'enseignement en lien avec le texte présenté. Ils sont donc familiarisés avec le domaine traité et avec l'informatique, largement utilisée lors des cours. Mais leur effectif étant trop faible, des personnes ne suivant pas l'unité d'enseignement sont également incluses. Ces dernières ne sont ni familiarisées avec le domaine du texte ni spécifiquement avec l'informatique.

Matériel. Chaque étudiant est placé devant un ordinateur et utilise l'application conçue pour l'expérience. Il leur est présenté successivement un questionnaire à choix multiples de quinze questions, un texte réparti sur huit pages (d'environ 310 mots chacune) parfois rattaché à une zone de texte pour la prise de notes, et de nouveau un questionnaire. Le texte porte sur le travail de l'enseignant et est issu d'un cours auquel est inscrit une partie des participants.

Procédure. Les étudiants sont répartis dans quatre groupes de 10 à 12 participants chacun. Deux groupes peuvent prendre des notes parmi lesquels un seul reçoit un feedback sur ses connaissances initiales du cours. Il en est de même pour les deux autres groupes ne prenant pas de notes. Le groupe témoin est celui qui ne prend pas de notes et qui ne reçoit pas de feedback. Cependant, l'activité des étudiants se déroule toujours en trois étapes, précédées d'un temps d'habituation, qui sont :

- Le pré-test: les étudiants sont tous évalués sur leurs connaissances du cours exposé, par quinze questions à choix multiples. Cependant, il est à noter que le score au pré-test est noté sur vingt-deux en raison du fait que plusieurs cases doivent être cochées à certaines questions. L'ensemble des questions concerne les idées générales de certains paragraphes du cours. Tous les paragraphes ne sont pas traités afin de ne pas lasser avec trop de questions. En revanche, chaque question se réfère à un paragraphe et un paragraphe ne peut faire l'objet que d'une seule question. Après chaque question, la moitié des étudiants reçoit un retour informatisé sur l'exactitude de leurs réponses sous la forme très bien, bien, moyen, mauvais ou très mauvais, selon le ratio de cases correctes cochées.

- La lecture du cours et la prise de notes : les étudiants accèdent au cours pendant 45 minutes. Le retour sur l'évaluation des connaissances initiales est transmis une seconde fois, face aux sections concernées du cours, aux étudiants des deux groupes feedback avec et sans prise de notes. Nous nous assurons ainsi que les effets observés ne sont pas liés à des différences de mémorisation de ce retour informatif. Par ailleurs, les étudiants de deux groupes sont confrontés à une interface où un espace est réservé à une prise de notes simultanée à la lecture (figure 1). Chaque page de cours correspond à une page de notes et le passage d'un écran à un autre n'autorise pas de retour. Les copier/coller sont verrouillés et la seule mise en page possible est le saut de lignes et le retrait par des espaces. Toutefois, aucune consigne concernant la prise de notes n'est donnée, sinon d'éviter les abréviations pour ne pas produire de biais dans l'analyse sémantique.

- Le post-test : les questions du pré-test sont présentées une nouvelle fois à tous les groupes, mais dans un ordre différent pour éviter toute habituation. Ce post-test est réalisé sans délai après la lecture et la prise de notes. En outre, plus aucun étudiant ne reçoit de feedback. 


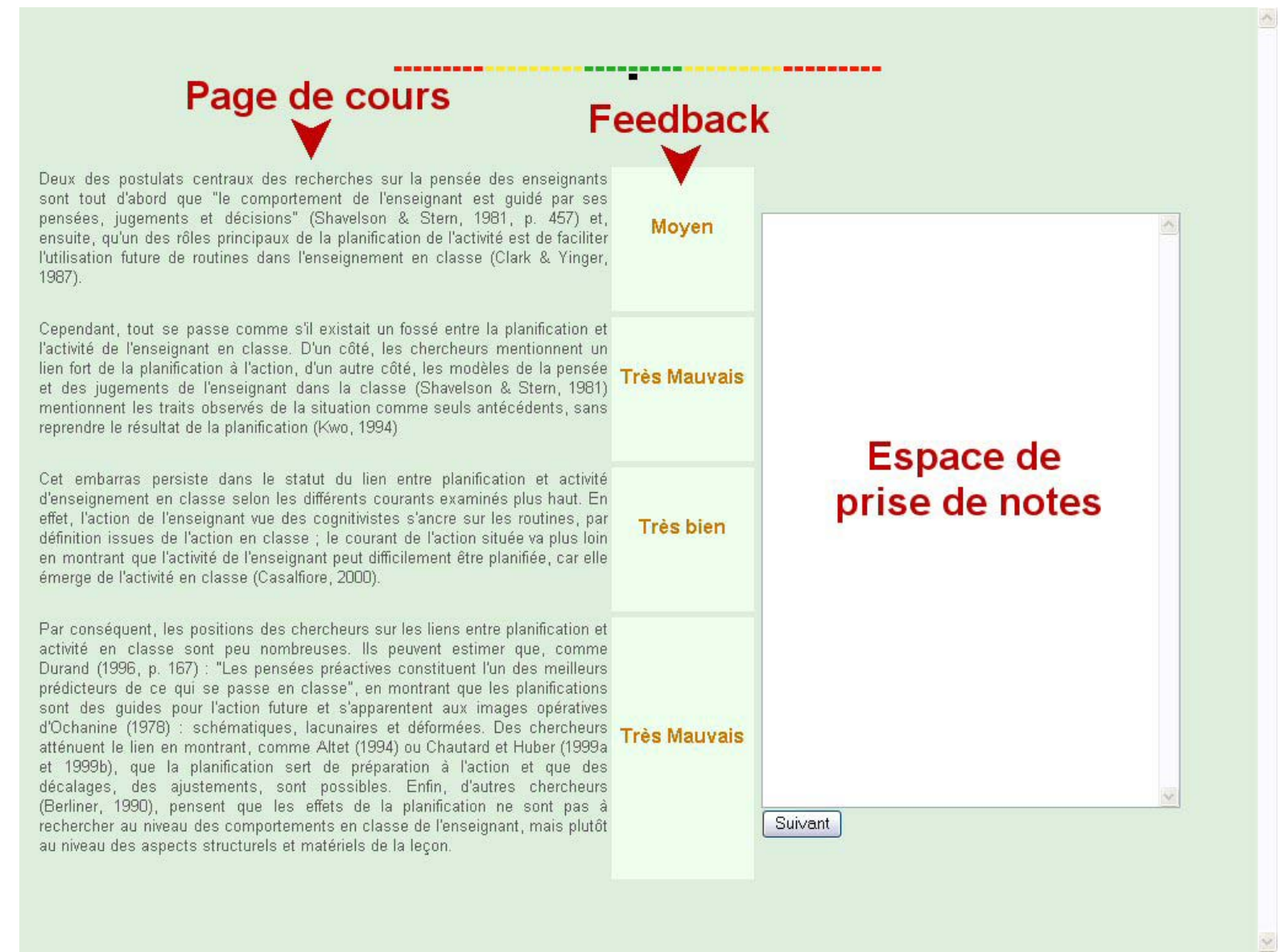

Figure 1 : Ecran d'une page de cours, du feedback et de l'espace de prise de notes

Traitement des données. Deux types de données sont recueillies : les scores aux questionnaires à choix multiples et les notes qui sont analysées avec LSA. Afin d'optimiser les calculs réalisés avec ce programme, nous utilisons un espace multidimensionnel que nous estimons acceptable, c'est-à-dire suffisamment représentatif des connaissances sémantiques des étudiants. Nous n'avons certes pas testé différents corpus pour conserver le plus optimal, mais nous en avons construit un en respectant au mieux les qualités prescrites dans la littérature. Il est donc volumineux [Dessus00] par l'intégration de l'ensemble des articles parus dans le journal Le Monde en 1999, et un minimum spécialisé [KurbyAl03] par la concaténation des articles à l'intégralité du cours dont est extrait le texte lu par les étudiants. La matrice construite sur ce corpus a été réduite à 300 dimensions $^{2}$. Trois valeurs sont ainsi dégagées :

- l'indice de cohérence des notes : la cohérence textuelle, telle qu'elle est mesurée, permet d'observer l'enchaînement des idées transcrites, phrase après phrase, par les étudiants. Elle correspond à la moyenne des indices de proximité sémantique calculés par LSA entre chaque phrase consécutive (phrase 1 et 2 , phrase 2 et $3, \ldots$ ) des huit pages de notes. Cette méthode de calcul reprend celle validée par Foltz, Kintsch et Landauer [FoltzA198] et par van Dijk et Kintsch (1983) [LandauerDumais97].

- l'indice de similitude de contenu partiel : la similitude de contenu partiel permet de vérifier si les étudiants se focalisent, lors de leur prise de notes, effectivement davantage sur les points correspondant à leurs lacunes. Elle est appréhendée par la moyenne des indices de proximité sémantique issus de la comparaison des notes à chaque section du cours non acquise. Les sections du cours sont considérées comme non acquises dès lors que les réponses initiales aux questions correspondantes posées correspondent à un feedback de valeur mauvais ou très mauvais, c'est-à-dire quand le nombre de cases correctement cochées est inférieur à la moitié du nombre de bonnes réponses attendues. 
- l'indice de similitude de contenu total : il est appréhendé par la moyenne des indices de proximité sémantique issus de la comparaison des notes à chaque section du cours, acquise ou pas. Cette valeur sert à vérifier que cette similitude de contenu produit bien un effet sur l'acquisition de nouvelles connaissances et que cet effet est augmenté par la présence d'un feedback.

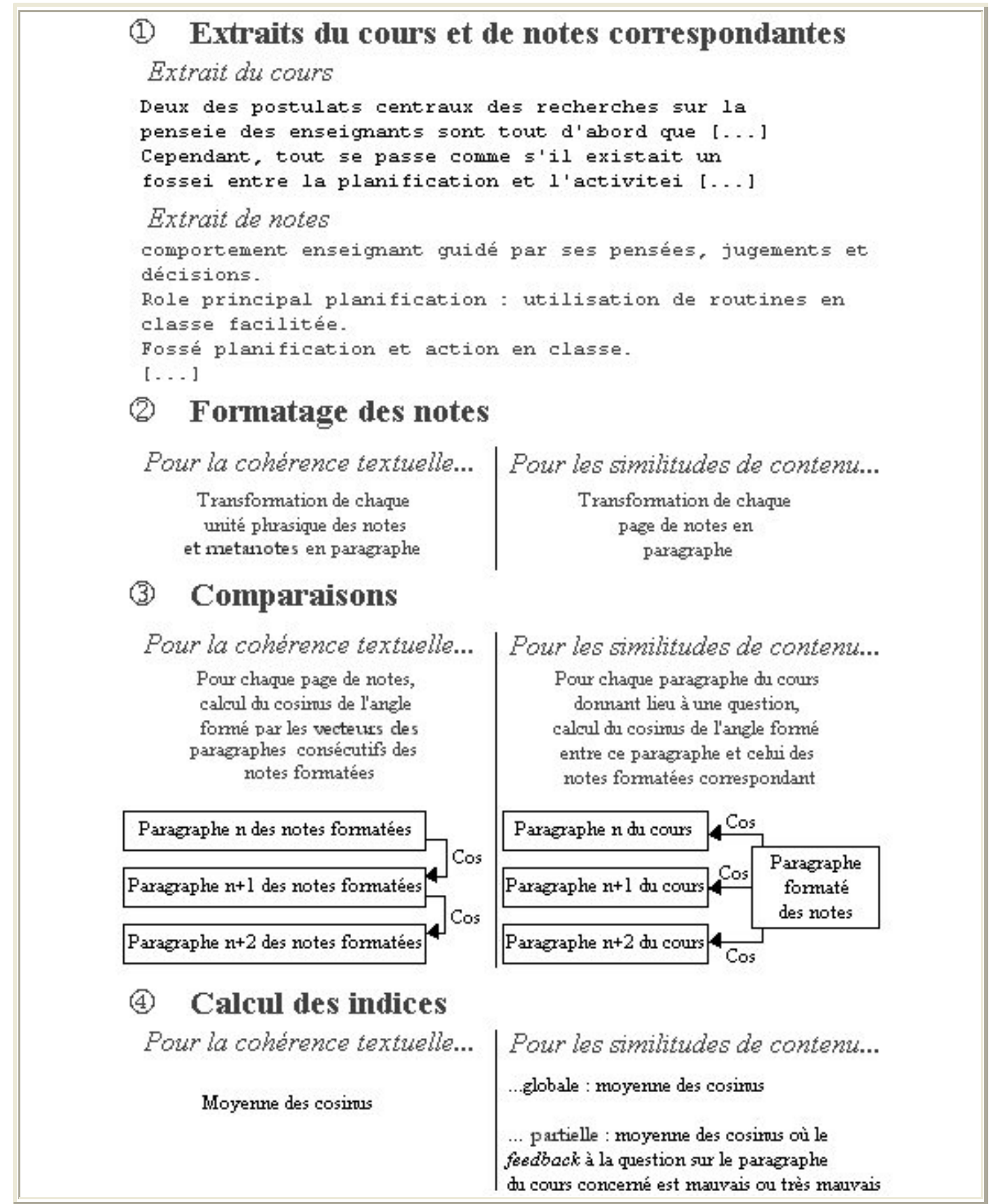

\section{Figure 2 : Calcul de la cohérence interphrases et calcul des similitudes de contenu à partir des notes d'un étudiant}

Ces calculs d'indices nécessitent toutefois que les notes subissent des mises en forme particulières (divisions ou regroupements des unités considérées en paragraphes). Les diverses mesures correspondent, ainsi, à des calculs de cosinus des angles formés par les vecteurs représentatifs des paragraphes. Notons aussi que nous ne supprimons aucune information. Si les participants ont inclus des méta-notes, elles sont traitées comme des phrases et chacune est donc transformée en paragraphe (figure 2). 


\subsection{Résultats}

\subsubsection{Analyse des progrès}

Une analyse des scores obtenus au pré-test montre que les différences observées parmi les groupes expérimentaux ne sont pas dues à des différences de répartition de la population mais bien au hasard. Nous pouvons donc considérer que les étudiants sont répartis équitablement $(F(3,40)=1,028 ; p=0,391)$. Les premiers tests statistiques montrent qu'une différence existe entre les progrès des étudiants de l'unité d'enseignement en lien avec le cours lu et les autres. Les premiers progressent moins que les seconds $(F(1,42)=6,643 ; p=0,014)$ sans qu'il existe pour autant d'effet plafond. Cette différence entre étudiants familiers et non familiers se retrouve dans les expériences de Peper et Mayer [PeperMayer86] en situation de prise de notes sur papier. Cependant, la différence qu'ils observent entre noteurs et non noteurs ne se retrouve pas dans nos résultats $(F(3,40)=0,579 ; p=0,632)$. La différence de progrès est donc sans doute liée à une différence très significative $(F(1,42)=5,316 ; p=0,026)$ (tableau 4$)$ dans les connaissances initiales entre étudiants familiers et non familiers au domaine du texte exposé.

Nous n'observons pas en revanche de différence significative entre les groupes recevant un feedback et entre les groupes n'en recevant pas $(F(1,42)=0,305 ; p=0,583)$. Donc, seule la distinction entre cours suivis permet de faire apparaître des différences de progrès. Nous ne pouvons malheureusement pas préciser l'effet de la prise de notes et du feedback spécifiquement sur ces groupes, l'effectif de l'échantillon étant trop faible ( $\underline{\text { tableau } 1)}$.

\begin{tabular}{|c|c|c|c|c|}
\hline & Feedback & Feedback + PDN & PDN & Contrôle \\
\hline Pré-test & $9,8(2,8)$ & $10,1(2,6)$ & $9,5(2,3)$ & $8,3(2,7)$ \\
\hline Post-test & $15,2(3,3)$ & $15,5(2,2)$ & $13,6(2,9)$ & $13,9(3,2)$ \\
\hline
\end{tabular}

Tableau 1 : Moyennes et écarts types du nombre de cases correctement cochées sur les 22 attendues aux pré et post-tests

\subsubsection{Analyse des notes}

L'effet du feedback n'est pas non plus évident sur la cohérence et les similitudes de contenu. Aucune différence significative n'apparaît (tableau 2). L'hypothèse formulée n'est donc pas vérifiée.

\begin{tabular}{|c|c|c|c|}
\hline & $\begin{array}{c}\text { Cohérence } \\
\text { interphrases }\end{array}$ & $\begin{array}{c}\text { Similitude de } \\
\text { contenu partiel }\end{array}$ & $\begin{array}{c}\text { Similitude de } \\
\text { contenu total }\end{array}$ \\
\hline Feedback+ PDN & $0,18(0,04)$ & $0,46(0,08)$ & $0,46(0,07)$ \\
\hline PDN & $0,17(0,05)$ & $0,41(0,10)$ & $0,42(0,10)$ \\
\hline
\end{tabular}

Tableau 2 : Moyennes et écarts types des cosinus obtenus en fonction du feedback

Concernant la similitude de contenu total (tableau 3), nous retrouvons également une différence significative entre les étudiants de l'unité d'enseignement concernée et les autres. Ceux de l'unité d'enseignement produisent des notes bien plus proches sémantiquement du texte lu que les autres $(F(1,19)=5,054 ; p=0,037)$. Leurs connaissances initiales plus importantes sur le domaine semblent donc leur permettre d'accéder plus facilement au contenu du cours. Cette différence est aussi présente lors de l'analyse de la similitude de contenu partiel $(F(1,19)=4,695 ; p=0,043)$.

Enfin, concernant la dimension de la cohérence interphrases (tableau 3), mesurée par LSA, nous observons, là encore, des différences significatives entre les étudiants des différentes unités d'enseignement. Lorsqu'ils ne reçoivent pas de feedback, les étudiants de l'unité d'enseignement sont plus 
cohérents que ceux ne le suivant pas $(F(1,8)=6,226 ; p=0,037)$. Mais la présence du feedback abaisse la cohérence interphrases des notes des étudiants suivant le cours $(F(1,8)=9,610 ; p=0,015)$ réduisant ainsi l'écart entre les deux populations. Ce phénomène se retrouve chez Boch [Boch99] dans ce qu'elle appelle la vision de l'aigle et de la tortue. Dans la vision de l'aigle, les étudiants familiers, qui ont davantage de connaissances initiales sur le cours, comprendraient mieux ce qu'ils lisent, augmentant ainsi le sens des notes transcrites et leur cohérence. A contrario, dès lors qu'ils reçoivent un feedback leur faisant prendre conscience de leurs connaissances initiales, ils acquièrent la vision de la tortue en ne cherchant à mémoriser que les nouvelles informations. Cette situation serait ici celle des étudiants non familiers du cours. Possédant de faibles connaissances sur le texte, ils auraient plus de difficultés à le comprendre. Leurs notes ne seraient alors qu'une énumération d'informations perçues.

\begin{tabular}{|c|c|c|c|c|c|}
\hline & Pré-test & Post-test & $\begin{array}{c}\text { Cohérence } \\
\text { interphrases }\end{array}$ & $\begin{array}{c}\text { Similitude de } \\
\text { contenu partiel }\end{array}$ & $\begin{array}{c}\text { Similitude de } \\
\text { contenu total }\end{array}$ \\
\hline $\begin{array}{c}\text { U.E. } \\
(\mathrm{n}=18)\end{array}$ & $10,4(2,87)$ & $14,3(3,50)$ & $0,18(0,024)$ & $0,48(0,082)$ & $0,48(0,080)$ \\
\hline $\begin{array}{c}\text { Hors U.E. } \\
(\mathrm{n}=26)\end{array}$ & $8,7(2,17)$ & $14,7(3,55)$ & $0,16(0,055)$ & $0,40(0,085)$ & $0,40(0,076)$ \\
\hline
\end{tabular}

Tableau 3 : Moyennes et écarts types des cosinus obtenus en fonction de l'U.E.

\subsection{Discussion}

Une absence de différence significative entre les groupes prenant des notes et n'en prenant pas s'observe ici sur un test à choix multiples. Toutefois, ce résultat ne signifie pas nécessairement que la prise de notes n'a eu aucun effet sur l'acquisition de connaissances. Effectivement, Peper et Mayer [PeperMayer86] ne trouvent un effet de la prise de notes que sur des tests d'application, et Peverly et al. [PeverlyA103] que sur des tests de rappel. Nous pouvons donc supposer qu'avec d'autres types de tests, nous aurions obtenu d'autres résultats.

Il ressort aussi que le feedback informatif, qui a pour but de faire prendre conscience aux étudiants de leurs connaissances initiales, ne modifie pas significativement les résultats au post-test. Peverly et al. [PeverlyA103] remarquent que la prise de notes permet à elle seule d'améliorer la connaissance des étudiants sur ce qu'ils savent et ce qu'ils ne savent pas. Le feedback que nous présentons ici peut donc n'être alors qu'un renforcement supplémentaire. Cependant, comme aucune différence significative n'apparaît parmi les groupes ne prenant pas de notes, cette hypothèse doit être rejetée. Nous admettons donc qu'un tel retour à un étudiant sur ses connaissances préalables ne favorise pas particulièrement les résultats à un test à choix multiples. Rappelons aussi que l'hypothèse qualitative posée n'est pas vérifiée : le feedback n'a donc pas non plus d'effet sur la cohérence interphrases et la similitude de contenu partiel des notes. Cet ensemble de résultats non attendus nous interroge sur la forme du feedback. Nous l'avons déterminé comme une appréciation basée sur une échelle à cinq niveaux. Or, le grand nombre d'appréciations possibles atténue probablement l'effet du feedback. Nous perdons l'opposition entre feedback positif et négatif. Peut-être qu'un simple feedback binaire tel que "correct" / "incorrect" aurait été préférable?

Des effets dus à l'appartenance à l'unité d'enseignement relative au cours exposé ont été relevés. D'une part, il y a un effet sur les connaissances finales qui est absorbé par la variable représentant les connaissances préalables. D'autre part, il y a un effet sur le contenu des notes tel que les étudiants inscrits à l'unité d'enseignement relative au texte exposé en produisent des plus proches du texte source et, en cas 
d'absence de feedback, des plus cohérentes. Cette dernière observation est cependant à mitiger dans la mesure où les effectifs des échantillons sont très faibles. Il peut toutefois être intéressant, dans une autre expérience sur l'impact d'un feedback informatif, d'analyser les différences d'effets produits en fonction de la familiarité des participants avec le domaine du texte exposé.

Cette étude permet donc de déterminer des facteurs explicatifs des scores au post-test par l'analyse sémantique des notes, mais aussi d'ouvrir plusieurs perspectives de recherches possibles dont l'une est de montrer qu'un tel feedback informatif peut agir différemment sur les individus selon leur familiarité avec le domaine des textes exposés.

\section{Remerciements}

Nous souhaitons remercier Patrick Mendelsohn pour l'aide qu'il a apportée dans cette recherche.

\section{Références}

Références bibliographiques

\section{[Baddeley93]}

Baddeley, A. (1993). La mémoire humaine: théorie et pratique. Grenoble : Presses Universitaires de Grenoble.

\section{[Beaufils01]}

Beaufils, A. (2001). Aide pour la recherche d'informations au collège et au lycée. In E. de Vries, J.-P. Pernin et J. P. Peyrin (Eds.), Actes du Cinquième Colloque Hypermédias et Apprentissages (pp. 167-181). Paris : INRP, EPI.

\section{[BlondelAl00]}

Blondel, F. M., Kempf, O., Schwob, M. (2000). Recherche d'informations sur la toile : pratiques d'élèves. Bulletin de l'Union des Physiciens, 828, 1819-1846.

\section{[Blondel01]}

Blondel, F. M. (2001). Pratiques documentaires en sciences avec Internet, outils de recherche et compétences des élèves. In W. Frindte, T. Köhler, P. Marquet, E. Nissen (Eds.), Actes du colloque INTELE 99 (pp. 73-78). Iena, Frankfort : Peter Lang.

\section{[Boch99]}

Boch, F. (1999). Pratiques d'écriture et de réécriture à l'université. La PDN, entre texte source et texte cible. Lille : Septentrion.

\section{[BrittonGulgoz91]}

Britton, B. K., Gulgoz, S. (1991). Using Kintsch's computational model to improve instructional text: Effects of repairing inference calls on recall and cognitive structures. Journal of Educational Psychology, 83(3), 329-345.

\section{[BrownSmiley78]}

Brown, A. L., Smiley, S. S. (1978). The development of strategies for studying texts. Child Development, 49, 1076-1088. 


\section{[Bruillard97]}

Bruillard, E. (1997). Les machines à enseigner. Paris : Hermès.

\section{[CanivetA186]}

Canivet, I., Lecocq, P., Ledru, C., Sizaire, P. (1986). La prise de notes : induction ou traitement ? Cahiers de Psychologie Cognitive, 6(3), 303-318.

\section{[Dansereau85]}

Dansereau, D. F. (1985). Learning strategy research. In J. W. Segal (Ed.), Thinking and learning skills (pp. 209-239). Hillsdale: Erlbaum.

\section{[Dessus00]}

Dessus, P. (2000). Construction de connaissances par exposition à un cours avec LSA. In Cognito, 18, $27-$ 34.

\section{[DessusAl00]}

Dessus, P., Lemaire, B., Vernier, A. (2000). Free-text assessment in a Virtual Campus. In K. Zreik (Ed.), Proc. International Conference on Human System Learning (CAPS'3) (pp. 61-76). Paris : Europia.

\section{[Fenouillet01]}

Fenouillet, F. (2001). La motivation chez les collégiens et les lycéens. In D. Gaonac'h, C. Golder (Eds.), Manuel de psychologie pour l'enseignant. Paris : Hachette Education.

\section{[FoltzA198]}

Foltz, P. W., Kintsch, W., Landauer, T. K. (1998). The measurement of textual coherence with Latent Semantic Analysis. Discourse processes, 25(2/3), 285-307.

\section{[GérouitA100]}

Gérouit, C., Roussey, J. Y., Barbier, M. L., Piolat, A. (2000). Prise de notes dans un environnement multimédia par des élèves de 10-12 ans plus ou moins bons lecteurs. Papier présenté à l'Atelier de Conjoncture de la SFP "Production et compréhension de documents techniques", Amiens, France.

\section{[GlenbergRobertson00]}

Glenberg, A. M., Robertson, D. A. (2000). Symbol grounding and meaning: A comparison of highdimensional and embodied theories of meaning. Journal of Memory and Language, 43, 379-401.

\section{[Hayes98]}

Hayes, J. R. (1998). Un nouveau cadre pour intégrer cognition et affect dans la rédaction. In A. Piolat, A. Pélissier (Eds.), La rédaction de textes : approche cognitive (pp. 51-101). Lausanne: Delachaux et Niestlé.

\section{[Kellogg98]}

Kellogg, R. T. (1998). Un modèle de la mémoire de travail dans la rédaction. In A. Piolat, A. Pélissier (Eds.), La rédaction de textes : approche cognitive (pp. 103-135). Lausanne : Delachaux et Niestlé.

\section{[KontostathisPottenger02]}

Kontostathis, A., Pottenger, W. M. (2002). Detecting patterns in the LSI term-term matrix. Papier présenté à la 2002 IEEE International Conference on Data Mining. 


\section{[KurbyA103]}

Kurby, A., Wiemer-Hastings, K., Ganduri, N., Magliano, J.P., Millis, K.K., McNamara, D. (2003). Computerizing reading training: Evaluation of a Latent Semantic Analysis space for science text. Behavior Research Methods, Instruments, and Computers, 35(2), 244-250.

\section{[LandauerDumais97]}

Landauer, T. K., Dumais, S. T. (1997). A solution to Plato's problem: The Latent Semantic Analysis theory of acquisition, induction and representation of knowledge. Psychological Review, 104, 211-240.

\section{[Landauer02]}

Landauer, T. K. (2002). On the computational basis of learning and cognition: Arguments from LSA. In N. Ross (Ed.), The Psychology of Learning and Motivation (Vol. 41, pp. 43-84). San Diego : Academic Press.

\section{[LehmanSchraw02]}

Lehman, S., Schraw, G. (2002). Effects of coherence and relevance on shallow and deep text processing. Journal of Educational Psychology, 94(4), 738-750.

\section{[LemaireDessus01]}

Lemaire, B., Dessus, P. (2001). A system to assess the Semantic Content of Student Essays. Journal of Educational Computing Research, 24(3), 305-320.

\section{[LemaireDessus03]}

Lemaire, B., Dessus, P. (2003). Modèles cognitifs issus de l'analyse de la sémantique latente. Cahiers Romans de Sciences Cognitives, 1, 55-74.

\section{[Leplat02]}

Leplat, J. (2002). Psychologie de la formation. Jalons et perspectives. Toulouse : Octarès Editions.

\section{[Mayer87]}

Mayer, R. E. (1987). Educational psychology, a cognitive approach. New York : Harper Collins.

\section{[McNamaraA196]}

Mc Namara, D., Kintsch, W., Butler Songer, N. (1996). Are good texts always better? Interactions of text coherence, background knowledge, and levels of understanding in learning from text. Cognition and Instruction, 14(1), 1-43.

\section{[OlivePiolat02]}

Olive, T., Piolat, A. (2002). Suppressing visual feedback in written composition: Effects on processing demands and coordination of the writing processes. International Journal of Psychology, 37(4), 209-218.

\section{[OxfordCrookall89]}

Oxford, R., Crookall, D. (1989). Research on language learning strategies: methods, findings, and instructional issues. Modern Language Journal, 73, 404-419.

\section{[PeperMayer86]}

Peper, R. J., Mayer, R. E. (1986). Generative effects of note-taking during science lectures. Journal of Educational Psychology, 78, 34-38. 


\section{[PeverlyAl03]}

Peverly, S. T., Brobst, K. E., Graham, M., Shaw, R. (2003). College adults are not good at self-regulation: A study on the relationship of self-regulation, note taking, and test taking. Journal of Educational Psychology, 95(2), 335-346.

\section{[Piolat01]}

Piolat, A. (2001). La prise de notes. Paris : Presses Universitaires de France.

\section{[RehderA198]}

Rehder, B., Schreiner, M. E., Wolfe, M. B. W., Laham, D., Landauer, T. K., Kintsch, W. (1998). Using Latent Semantic Analysis to assess knowledge: Some technical considerations. Discourse Processes, 25, 309-336.

\section{[Reuter94]}

Reuter, Y. (Ed.). (1994). Les interactions lecture-écriture, actes du colloque Theodile-Crel'93. Berne : Peter Lang.

\section{[Romainville93]}

Romainville, M. (1993). Savoir parler de ses méthodes : métacognition et performance à l'université. Bruxelles : De Boeck Université.

\section{[SimonetSimonet97]}

Simonet, R., Simonet, J. (1997). La prise de notes intelligente. Paris : Les Editions d'Organisation.

\section{[TricotA198]}

Tricot, A., Pierre-Demarcy, C., El Boussarghini, R. (1998). Un panorama des recherches consacrées à l'étude de l'activité mentale de l'utilisateur d'un hypermédia. Sciences et Techniques Educatives, 5(4), 371-400.

\section{[WolfeAl98]}

Wolfe, M. B. W., Schreiner, M. E., Rehder, B., Laham, D., Foltz, P. W., Kintsch, W. (1998). Learning from text: Matching readers and texts by Latent Semantic Analysis. Discourse Processes, 25, 309-336.

\section{A propos des auteurs :}

Sonia MANDIN est doctorante à l'Université Pierre-Mendès-France de Grenoble, France. Elle prépare une thèse sur la modélisation et la simulation de l'activité de résumé de textes, ainsi que son instrumentalisation sous la forme d'un tuteur informatique d'aide au résumé.

Adresse : Laboratoire des Sciences de l'Education - Université Pierre-Mendes-France, BP 47 F-38040 Grenoble Cedex 9

\section{Courriel : $\underline{\text { Sonia.Mandin@upmf-grenoble.fr }}$}

Toile : http://www.upmf-grenoble.fr/sciedu/smandin/

Philippe DESSUS est maître de conférences en sciences de l'éducation et chercheur au Laboratoire des Sciences de l'Éducation de Grenoble. Il s'intéresse aux modélisations cognitives basées sur l'analyse de la sémantique latente. 
Revue sticef.org

Adresse : Laboratoire des Sciences de 1'Education - Université Pierre-Mendes-France, BP 47 F-38040 Grenoble Cedex 9

Courriel : $\underline{\text { Philippe.Dessus@upmf-grenoble.fr }}$

Toile : http://www.upmf-grenoble.fr/sciedu/pdessus/

Benoît LEMAIRE est maître de conférences en informatique et chercheur au Laboratoire Leibniz de l'IMAG. Il s'intéresse aux modélisations cognitives basées sur l'analyse de la sémantique latente.

Adresse : Institut d'Informatique et de Mathématiques Appliquées de Grenoble (IMAG), Laboratoire LEIBNIZ-IMAG (UMR CNRS 5522), 46 avenue Félix Viallet, 38031 Grenoble Cedex 1

Courriel : Benoit.Lemaire@,imag.fr

Toile : http://www.upmf-grenoble.fr/sciedu/blemaire/

[1]. Un texte est rendu cohérent en augmentant, dans chaque phrase, le nombre de répétitions de mots présents dans les propositions précédentes.

[2]. 300 facteurs, 214685 termes et 511678 paragraphes

\section{Référence de l'article :}

Benoît LEMAIRE, Philippe DESSUS, Benoît LEMAIRE, Effet d'un feedback informatif sur la prise de notes dans un environnement d'apprentissage informatisé, Revue STICEF, Volume 12, 2005, ISSN : 1764-7223, mis en ligne le 15/05/2005, http://sticef.org

(C) Revue Sciences et Technologies de 1'Information et de la Communication pour l'Éducation et la Formation, 2005 